\title{
Elastic strain and dopant activation in ion implanted strained Si nanowires
}

R. A. Minamisawa, S. Habicht, D. Buca, R. Carius, S. Trellenkamp, K. K. Bourdelle, and S. Mantl

Citation: Journal of Applied Physics 108, 124908 (2010);

View online: https://doi.org/10.1063/1.3520665

View Table of Contents: http://aip.scitation.org/toc/jap/108/12

Published by the American Institute of Physics

\section{Articles you may be interested in}

Band structure, deformation potentials, and carrier mobility in strained $\mathrm{Si}, \mathrm{Ge}$, and $\mathrm{SiGe}$ alloys Journal of Applied Physics 80, 2234 (1998); 10.1063/1.363052

The physics and chemistry of the Schottky barrier height

Applied Physics Reviews 1, 011304 (2014); 10.1063/1.4858400

Evidence for strong Fermi-level pinning due to metal-induced gap states at metal/germanium interface Applied Physics Letters 91, 123123 (2007); 10.1063/1.2789701

Fermi-level pinning and charge neutrality level in germanium

Applied Physics Letters 89, 252110 (2006); 10.1063/1.2410241

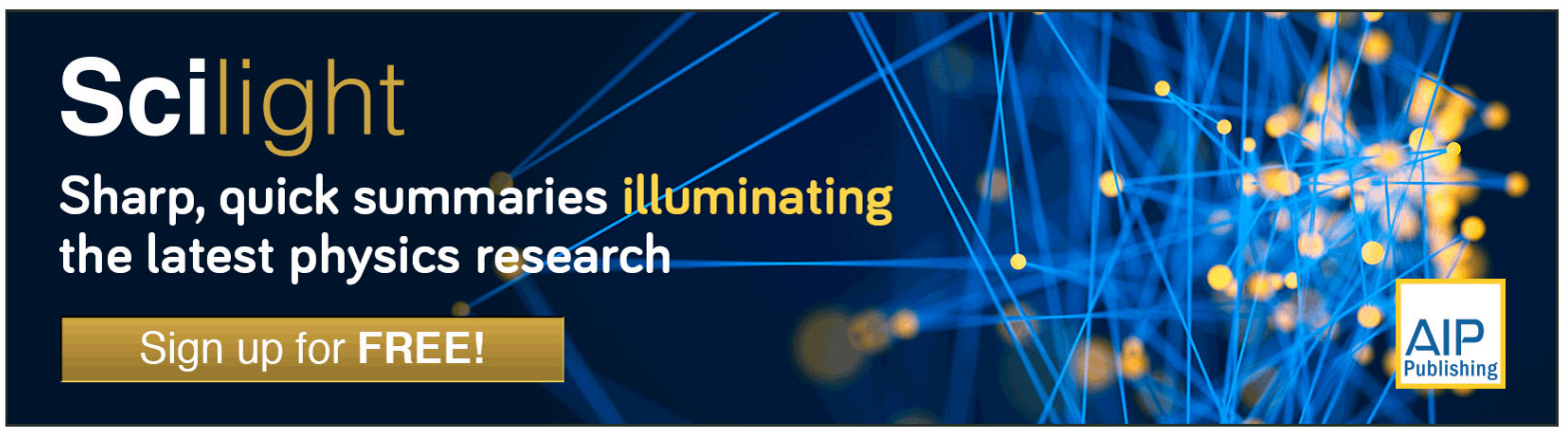




\title{
Elastic strain and dopant activation in ion implanted strained Si nanowires
}

\author{
R. A. Minamisawa, ${ }^{1, a)}$ S. Habicht, ${ }^{1}$ D. Buca, ${ }^{1}$ R. Carius,${ }^{2}$ S. Trellenkamp,${ }^{1}$ K. K. Bourdelle, ${ }^{3}$ \\ and S. Manti ${ }^{1}$ \\ ${ }^{1}$ JARA-Fundamentals of Future Information Technology, Forschungszentrum Jülich, 52425 Jülich, \\ Germany \\ ${ }^{2}$ Institute of Energy Research (IEF), Forschungszentrum Jülich, 52425 Jülich, Germany \\ ${ }^{3}$ SOITEC, Parc Technologique des Fontaines, 38190 Bernin, France
}

(Received 3 May 2010; accepted 27 October 2010; published online 23 December 2010)

\begin{abstract}
Strained Si nanowires (NWs) are attractive for deeply-scaled complementary metal-oxidesemiconductor devices due to the combination of enhanced carrier mobility and excellent electrostatic control as was demonstrated with trigate metal-oxide-semiconductor field effect transistors. The challenge in using strained Si NWs for devices is to preserve the elastic strain during the required processing steps. In this work we investigated the influence of fundamental processing steps like patterning and dopant ion implantation on the structural and transport properties of strained Si layers and NWs on silicon-on-insulator (SOI) substrates. NWs with widths down to 35 $\mathrm{nm}$, fabricated on $25 \mathrm{~nm}$ strained SOI and implanted to doses ranging from $5 \times 10^{14}$ to 2 $\times 10^{15}$ ions $/ \mathrm{cm}^{2}$ were investigated. We show that strain conservation and a low sheet resistivity of $6.2 \times 10^{-4} \Omega \mathrm{cm}$, close to the layer resistivity, can only be obtained if the NWs are patterned on doped layers. For NWs directly implanted to doses above $1 \times 10^{15}$ ions $/ \mathrm{cm}^{2}$, complete strain relaxation and structural disorder by solid phase recrystallization were observed. In both cases, NWs with widths smaller than $55 \mathrm{~nm}$ exhibit an increased specific resistivity. (C) 2010 American Institute of Physics. [doi:10.1063/1.3520665]
\end{abstract}

\section{INTRODUCTION}

Advances in nanotechnology have empowered the development of innovative devices, with rapidly increasing packing density and functional complexity. The small size and excellent material properties of nanowires (NWs) have led to the demonstration of NW-based electronic, optical, and bio/ environmental sensing devices. ${ }^{1}$ However, in the past most NW based devices investigated were fabricated using a bottom up approach, such as the vapor-liquid-solid growth method $^{2}$ with nanoparticles as catalysts. The main problem of bottom up grown NWs relies in the positioning and alignment of the nanostructures to electrodes, contacts, and control gates, indispensable for the realization of active devices such as field effect transistors (FETs).

A top down approach simplifies fabrication and processing of NWs since standard integrated circuit technology, without positioning and/or alignment issues, can be used. Moreover, performance boosters such as strained and/or alternative channel materials may be employed to improve carrier transport, essentially for future complementary metaloxide-semiconductor technologies. Strained $\mathrm{Si}$ is a well established material with enhanced carrier mobilities due to strain-induced changes in conduction and valence bands by band splitting and/or band warping. ${ }^{3}$ Strained channels were successfully transferred to the NW architecture concept using local stressor technologies ${ }^{4}$ or strained silicon-oninsulator (SSOI) wafers, ${ }^{5}$ providing simultaneously enhanced carrier mobilities, better electrostatic control, and low power consumption. ${ }^{6}$

${ }^{a)}$ Electronic mail: r.a.minamisawa@fz-juelich.de.
Ion implantation is a standard doping technique in semiconductor manufacturing, e.g., for source and drain (S/D) fabrication. High performance bottom-up ${ }^{7}$ and top-down $\mathrm{Si}$ NWs FETs with ohmic contacts fabricated by ion implantation have been reported. Additionally, a new type of transistor using heavily doped silicon NWs was demonstrated, where the doping concentration in the channel is identical to that in $\mathrm{S} / \mathrm{D} .^{8}$

Although SSOI layers are thermally stable up to $1000{ }^{\circ} \mathrm{C}$ annealing, ${ }^{9}$ nanostructure patterning and ion implantation may drastically modify the strain state. ${ }^{10}$ Baudot et al. ${ }^{11}$ have shown that short $(<200 \mathrm{~nm})$ but wide $(4 \mathrm{~mm})$ channels of planar SSOI devices relax up to $80 \%$ along the current transport direction during high dose ion implantation of adjacent S/D regions. Therefore, optimization of S/D implantation parameters becomes a critical step for increased electrical performance of devices using strained layers.

As a consequence, comprehensive understanding of structural, mechanical and electrical properties of ion implanted strained Si NWs is required. So far, several articles reported on ion beam doped strained Si NWs and fabrication of devices; ${ }^{6,9,12}$ however, a systematic analysis assessing fundamental material aspects such us recrystallization, strain recovery and doping activation of implanted strained Si NWs would be of extreme benefit.

In this work we present a detailed study of the effect of $\mathrm{As}^{+}$ion implantation and spike annealing on SSOI layers and strained NWs. Three principal cases are analyzed: (i) the influence of the ion implantation on the layer and NW crystallinity, (ii) the strain relaxation of the implanted structures, and (iii) the resistivity of the structures as a measure for dopant activation. 


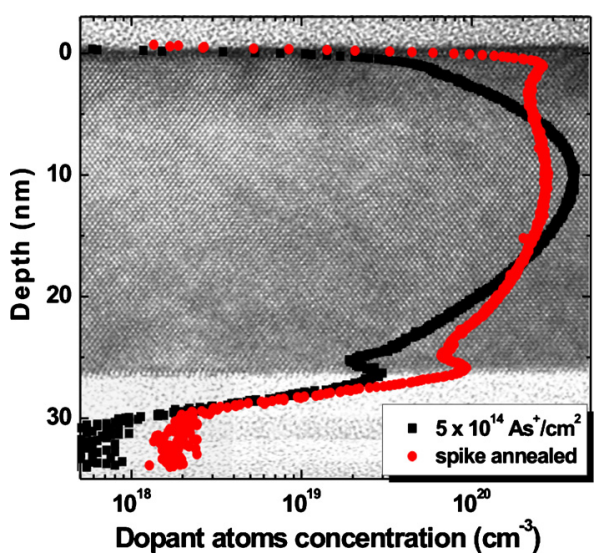

FIG. 1. (Color online) SIMS profiles of $25 \mathrm{~nm}$ SSOI layers implanted with $5 \times 10^{14} \mathrm{As}^{+} / \mathrm{cm}^{2}$ : as-implanted (squares) and $1000{ }^{\circ} \mathrm{C}$ spike annealed (circles) superposed on a XTEM image of the SSOI wafer.

In summary, we devote this article to the concept of formation of doped strained Si NWs which is not only restricted to our specific experimental conditions but contributes to a more general understanding of strained layer relaxation.

\section{EXPERIMENTAL}

The starting material for the NW fabrication consisted of $25 \mathrm{~nm}$ thick biaxial tensile strained SOI layer on top of a 145 $\mathrm{nm}$ buried oxide (BOX) layer. Details on the fabrication process and the properties of the SSOI material are described elsewhere. ${ }^{13}$ Raman and x-ray diffraction measurements indicated a biaxial tensile strain of $\varepsilon=0.8 \%$ in the SSOI layer.

Implantation of $\mathrm{As}^{+}$was carried out at an energy of 10 $\mathrm{keV}$ to doses ranging from $5 \times 10^{14}$ to $2 \times 10^{15} \mathrm{ions} / \mathrm{cm}^{2}$, which are high enough to induce a phase transition from crystalline to amorphous within the implanted region. In order to avoid channeling the ion implantation was carried out at $7^{\circ}$ tilt. The projected ion range of $12.5 \mathrm{~nm}$, as calculated using SRIM 2008 simulation software, ${ }^{14}$ is located close to the center of the SSOI layer, therefore, assuring a quasisymmetrical in-depth As dopant distribution after annealing. Dopant activation was obtained by spike annealing at $1000{ }^{\circ} \mathrm{C}$ in nitrogen atmosphere using a rapid thermal processing system. Spike annealing suppresses dopant diffusion largely, forming a sharp doping profile as required for short channel and tunneling FET devices. ${ }^{15}$ Figure 1 displays secondary ion mass spectroscopy (SIMS) depth profiles of asimplanted (squares) and annealed (circles) samples superposed on a cross-section transmission electron microscopy (TEM) image of a SSOI layer prior to ion implantation. The increased concentration of $\mathrm{As}$ at the $\mathrm{Si} / \mathrm{BOX}$ interface is a SIMS artifact due to different sputtering rates of the strained $\mathrm{Si}$ and $\mathrm{SiO}_{2}$ layers.

Electron beam lithography (EBL) and reactive ion etching using low energy fluorine based plasma were employed to define NWs with widths ranging from 35 to $75 \mathrm{~nm}$, oriented along the [110] crystal direction. The NW dimensions were checked by scanning electron microscopy (SEM). Specific NW structures were fabricated to optimize the investigation of recrystallization, strain recovery, and doping acti-

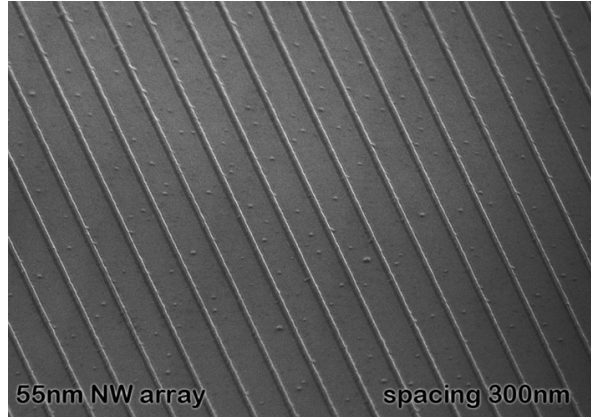

(a)

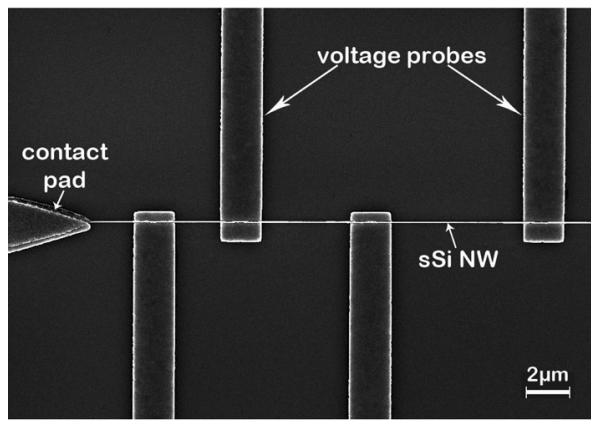

(b)

FIG. 2. SEM image of (a) a dense array of patterned SSOI NWs used for the Raman measurements and (b) TLM structure used for the electrical characterization of the doped NWs.

vation of implanted strained $\mathrm{Si}$ NWs but maintaining relevant dimensions constant for comparison.

Raman spectroscopy in backscattering geometry is a useful nondestructive tool to analyze mechanical stress and composition in semiconductor materials. ${ }^{16,17}$ For thin layer, and especially for the case of structured layers, the strong Raman signal from the Si substrate makes it difficult to extract the strained layer signal. In order to overcome this problem, $30^{\circ}$ off-axes geometry was employed which allowed a better light coupling and, if necessary, the use of a light polarizer and/or analyzer. The structures processed for strain measurements by Raman spectroscopy consisted of arrays of $600 \mu \mathrm{m}$ long NWs with $300 \mathrm{~nm}$ pitch size. These arrays enable the detection of a strong Raman intensity with a high signal to noise ratio. ${ }^{18}$ We used a low power laser density to avoid heating induced relaxation of the NWs. The resolution of the Raman setup was $0.5 \mathrm{~cm}^{-1}$. Figure 2(a) presents a SEM image of a $55 \mathrm{~nm}$ narrow NW array.

The resistivity of the NWs was extracted using the transmission line model (TLM). ${ }^{19}$ For these measurements, long NWs with similar widths as for the Raman structures but with contact pads at their extremities, were fabricated in order to assure equivalent strain state. The metal Au/Cr (100 $\mathrm{nm} / 20 \mathrm{~nm}$ ) electrode contacts were defined by EBL and liftoff process. Figure 2(b) shows the SEM image of a TLM structure with $35 \mathrm{~nm}$ wide wires. The electrical properties of the NWs were measured using a standard probe station coupled to a Keithley parameter analyzer. Details on the resistivity extraction method are described elsewhere. ${ }^{19}$ The resistivity of the doped SSOI layers was measured using the van der Pauw method. 


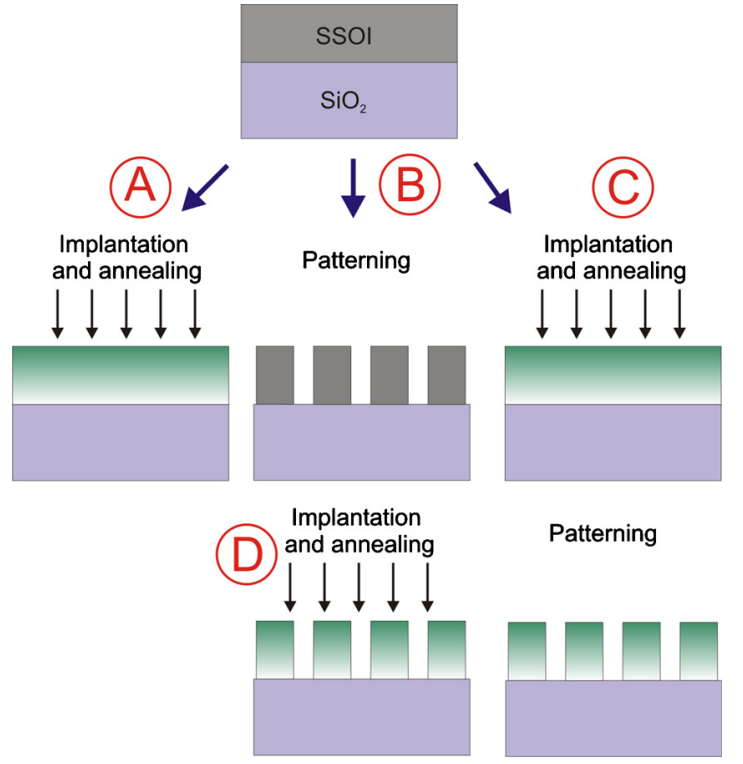

FIG. 3. (Color online) Illustration of the processing sequence and the following four cases studied: (a) implanted and annealed biaxially tensile layers of SSOI; (b) patterned unimplanted SSOI layers; (c) NWs patterned on implanted and annealed SSOI layers, and (d) patterned NWs followed by implantation and annealing.

\section{RESULTS AND DISCUSSION}

In order to discuss the experiments in a systematic manner, the results on implanted and/or patterned samples are presented in the following sequence: (a) implanted and annealed biaxial tensile SSOI layers; (b) patterned unimplanted SSOI layers; (c) NWs patterned on implanted and annealed SSOI layers, and (d) patterned NWs followed by implantation and annealing. Figure 3 schematically illustrates the process flow.

\section{A. $\mathrm{As}^{+}$implantation of SSOI layers}

The implantation of heavier ions at high doses into $\mathrm{Si}$ is known to result in the formation of defective regions or even amorphization. Intensive studies were dedicated to the thermal evolution of such implantation induced defect layers and to structural changes. Most of this defect research was related to transient enhanced diffusion of dopants ${ }^{20}$ but also to strain relaxation of thin layers. ${ }^{21}$ In SOI, layer recrystallization occurs only if a crystalline seed layer remains after implantation. Recently, it was shown that full strain recovery can be obtained by solid phase epitaxial regrowth (SPER) of partially amorphized SSOI. ${ }^{22}$

The amorphization of a layer occurs when the vacancy concentration (Frenkel pair concentration) exceeds a certain critical value. Therefore, higher implantation doses result in thicker amorphous layers, and consequently, in a thinner strained Si seed layer available for recrystallization. Within the accuracy of the SRIM simulations, we estimated the amorphous layer thickness for the implantation doses used. Figure 4(a) presents the vacancy distribution profiles in $25 \mathrm{~nm}$ SSOI layers after $10 \mathrm{keV} \mathrm{As}^{+}$ion implantation to doses of 5 $\times 10^{14} \mathrm{~cm}^{-2}$ (squares), $1 \times 10^{15} \mathrm{~cm}^{-2}$ (circles), and 2 $\times 10^{15} \mathrm{~cm}^{-2}$ (triangles) doses. The thickness of the amorphized Si layer is taken at the critical vacancy concentration
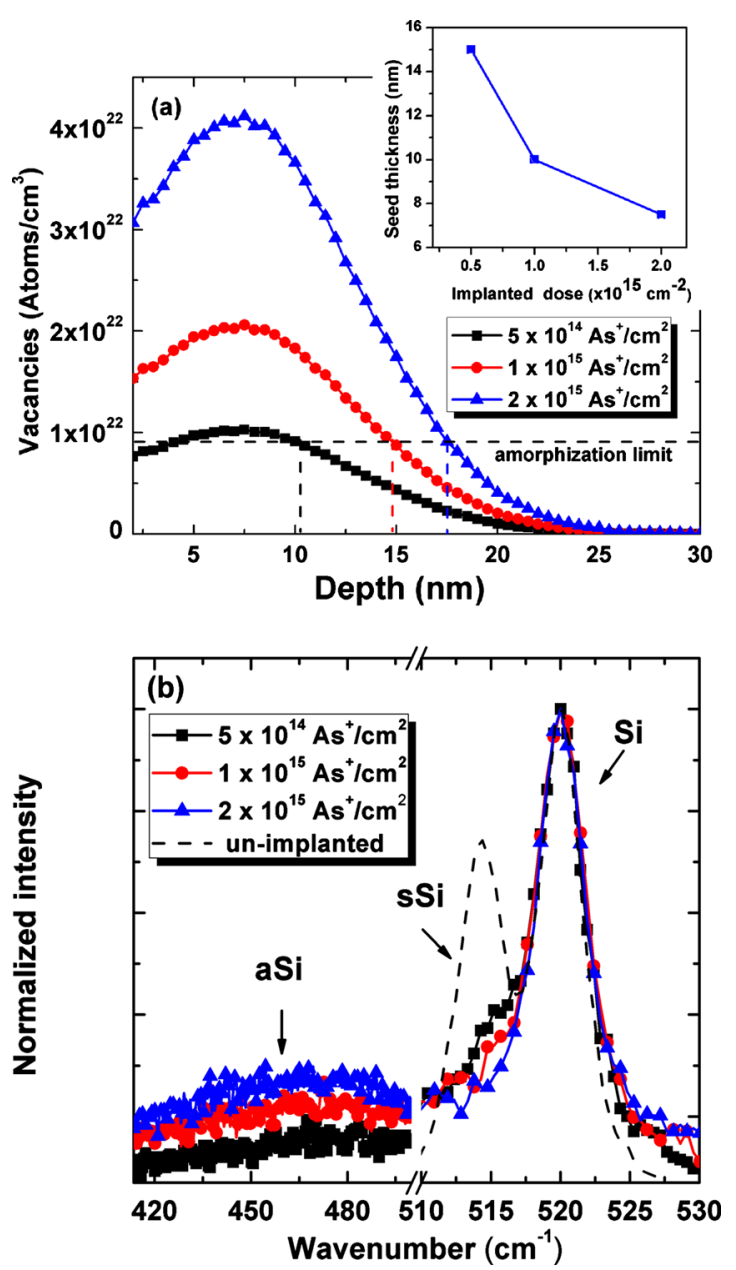

FIG. 4. (Color online) (a) Profiles of vacancy distribution in $25 \mathrm{~nm}$ SSOI layer after ion implantation of $5 \times 10^{14}$ (squares), $1 \times 10^{15}$ (circles), and 2 $\times 10^{15} \mathrm{As}^{+} / \mathrm{cm}^{2}$ (triangles). The black dashed line represents the amorphization threshold. The inset shows the thickness of the seed layer vs the implantation dose. (b) Raman spectra of unimplanted and as-implanted SSOI layers indicating a decrease in the strained Si signal and an increase in the amorphous Si signal with the implantation dose.

value of $18 \%$ of the $\mathrm{Si}$ atomic density, corresponding to $8.95 \times 10^{21} \mathrm{~cm}^{-3} \cdot{ }^{21}$

Figure 4(b) displays the Raman spectra of the unimplanted and as-implanted SSOI layers for different $\mathrm{As}^{+}$ion implanted doses. The peak at $520 \mathrm{~cm}^{-1}$ corresponds to bulk cubic $\mathrm{Si}$, while the second peak, located at $514.5 \mathrm{~cm}^{-1}$, represents the $0.8 \%$ tensile strained Si layer. The increase in the intensity of the amorphous layer signal, the broad peak centered around $480 \mathrm{~cm}^{-1}$, and the decrease of the strained $\mathrm{Si}$ signal with implantation dose confirm the SRIM simulation results of increased layer amorphization with the implantation doses. After spike annealing, the amorphous-Si peaks vanish and the strained Si peaks recover greatly with a slight asymmetrical broadening and a minor redshift [Fig. 5(a)]. Mostly, a shift in Si-Si Raman phonon mode, observed in implanted samples, is attributed to an increase in tensile strain. However, Cerdeira and Cardona ${ }^{23}$ studied the dependence of the first-order Raman frequency of $\mathrm{Si}$ on carrier concentration $N_{\mathrm{a}}$ (per cubic centimeter), finding that doping alters the lattice deformation potential of $\mathrm{Si}$, resulting in an effective softening of the lattice and giving lower phonon 

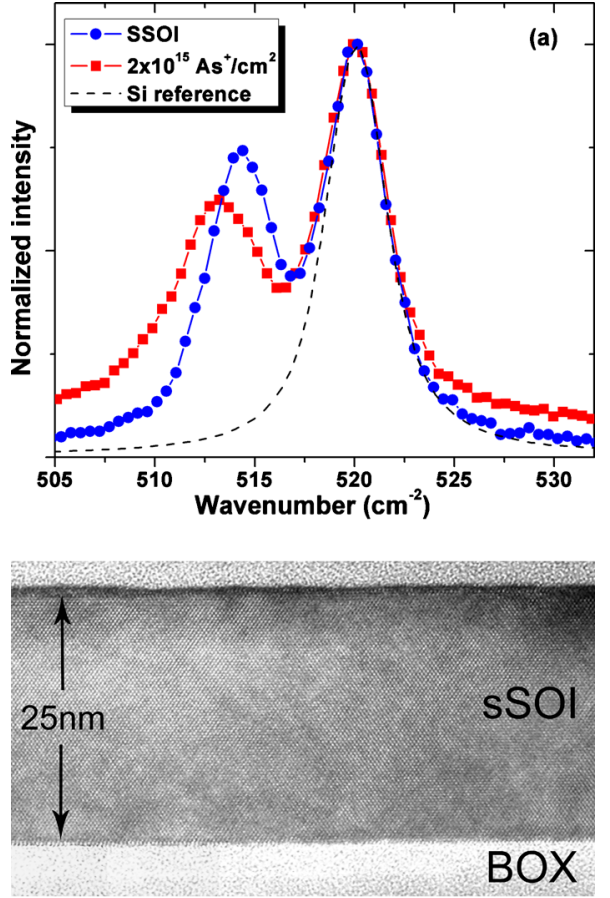

(b)

FIG. 5. (Color online) (a) Raman spectra of SSOI layers unimplanted (circles) and after $2 \times 10^{15} \mathrm{As}^{+} / \mathrm{cm}^{2}$ implantation and spike annealing (squares); (b) the XTEM image of this sample shows a defect free recrystallization even if only $6 \mathrm{~nm}$ seed layer is available.

vibration frequencies (Raman redshifts) at high carrier concentrations. Similar observations were also reported by O'Reilly et al. ${ }^{24}$ and Heiermann et al. ${ }^{22}$ on Sb implanted SSOI layers. High resolution $\mathrm{x}$-ray diffraction measurements (not shown here) confirmed that the lattice constant of the implanted and annealed SSOI layer is identical with the unimplanted structures. This observation indicates that layer recrystallization is indeed epitaxial, and even more important, pseudomorphic. Therefore, the Raman shift in $\mathrm{Si}-\mathrm{Si}$ mode of the doped samples is attributed to doping effect. The cross-section transmission electron microscope (XTEM) image Fig. 5(b) confirms that the strained Si layer is single crystalline and defect free, even if regrowth starts from a 7 $\mathrm{nm}$ strained $\mathrm{Si}$ seed layer only, as in the case of the 2 $\times 10^{15} \mathrm{As}^{+} / \mathrm{cm}^{2}$ implantation dose.

The sheet resistance of the implanted layers was measured using a four-point van der Pauw method and, in combination with Hall-measurements, the carrier-concentration, and Hall-mobility were determined. Table I displays the measured values for layer resistivity, carrier mobility, and con-

TABLE I. Carrier mobility, concentration and resistivity of doped for $25 \mathrm{~nm}$ SSOI layers for different $\mathrm{As}^{+}$implantation doses and $1000{ }^{\circ} \mathrm{C}$ spike annealing.

\begin{tabular}{cccc}
\hline \hline $\begin{array}{c}\text { Implantation } \\
\text { dose } \Phi \\
\left(\mathrm{As}^{+} / \mathrm{cm}^{2}\right)\end{array}$ & $\begin{array}{c}\text { Electron } \\
\text { mobility } \mu_{0} \\
\left(\mathrm{~cm}^{2} / \mathrm{V} \mathrm{s}\right)\end{array}$ & $\begin{array}{c}\text { Dopant } \\
\text { conc. } N_{\mathrm{a}-\mathrm{L}} \\
\left(\mathrm{cm}^{-3}\right)\end{array}$ & $\begin{array}{c}\text { Resistivity } \\
\rho \\
(\Omega \mathrm{cm})\end{array}$ \\
\hline $5 \times 10^{14}$ & 77 & $6.5 \times 10^{19}$ & $1.2 \times 10^{-3}$ \\
$1 \times 10^{15}$ & 60.5 & $1.2 \times 10^{20}$ & $7.5 \times 10^{-4}$ \\
$2 \times 10^{15}$ & 47 & $1.9 \times 10^{20}$ & $6.2 \times 10^{-4}$ \\
\hline \hline
\end{tabular}

centration of activated As atoms. The layer resistivity decreases from $1.2 \times 10^{-3}$ to $6.3 \times 10^{-4} \Omega \mathrm{cm}$ as the implantation doses increases from $5 \times 10^{14}$ to 2 $\times 10^{15} \mathrm{~cm}^{-2}$, reaching an active dopant concentration of $1.9 \times 10^{20} \mathrm{~cm}^{-3}$, which corresponds to the As solubility limit in $\mathrm{Si}$ of $2 \times 10^{20} \mathrm{~cm}^{-3}$. However, as expected for highly doped layers, the carrier concentration increases with the implanted dose while the mobility degrades as a consequence of increased Coulomb scattering.

In conclusion, highly doped and fully strained Si layers are obtained after implantation and annealing at $1000{ }^{\circ} \mathrm{C}$, even if only an ultrathin strained Si seed is available for layer recrystallization. These results serve as reference for the following experiments on patterned Si NWs. Before presenting and discussing the ion implantation effects on strained NWs the effect of patterning on strain relaxation is studied.

\section{B. Patterning of unimplanted strained Si layers}

Since the $90 \mathrm{~nm}$ technology node when strain-induced mobility enhancement first entered manufacturing, strain measurements have become mandatory for front-end processes. Particularly, measurement of the elastic strain in asymmetric nanostructures has become vital for the optimization of strained Si-based devices and for the development of quantitative simulation models. In case of NWs, it has been shown that elastic relaxation strongly depends on the NW dimensions. ${ }^{6}$ Simulations indicate that the remaining strain within the NWs concentrates near the $\mathrm{Si} / \mathrm{SiO}_{2}$ interface region and becomes uniform with decreasing SSOI layer thickness. ${ }^{18,25}$ On the other hand, patterning of SSOI layers to convert biaxial tensile strain into uniaxial tensile strain led to high performance strained $\mathrm{Si}$ NW metal-oxidesemiconductor FETs. ${ }^{26-28}$

Figure 6(a) presents the Raman spectra of NW arrays with different widths. The strained Si peak shifts toward the cubic-Si position with decreasing NW width, indicating elastic strain relaxation. ${ }^{18}$ The exact peak positions were determined by deconvolution of the strained and cubic Si signals. Unfortunately, due to the geometry employed in this work, the Raman signal corresponds to the sum of the in-plane strain components, and cannot be directly decoupled in the measurement. The Raman peak shift, $\Delta \omega$, correlates with the elastic strain in Si as follows: ${ }^{29}$

$$
\Delta \omega=-1.92 \mathrm{~cm}^{-1} \times 180\left(\varepsilon_{x x}+\varepsilon_{y y}\right),
$$

where $\varepsilon_{x x}$ and $\varepsilon_{y y}$ are the strain across and along the NW, respectively. By assuming negligible relaxation along the NWs,${ }^{11,18}$ we can estimate the $\varepsilon_{x x}$ strain component across the NWs. Figure 6(b) shows $\varepsilon_{x x}$ as a function of NW width for different SSOI layer thicknesses with same initial biaxial strain in the Si layer of $\varepsilon \sim 0.8 \%$. In this comparison, the SSOI thickness varies from 10 to $70 \mathrm{~nm}$. Our results for the $25 \mathrm{~nm}$ thick SSOI NW are consistent with the Raman measurement of Lei et al. for a NW array fabricated on $30 \mathrm{~nm}$ SSOI. ${ }^{18}$ The results from Ref. 11 were added to emphasize that patterning of strained Si layers leads to strain relaxation, which is more pronounced if thicker SSOI layers are used. 

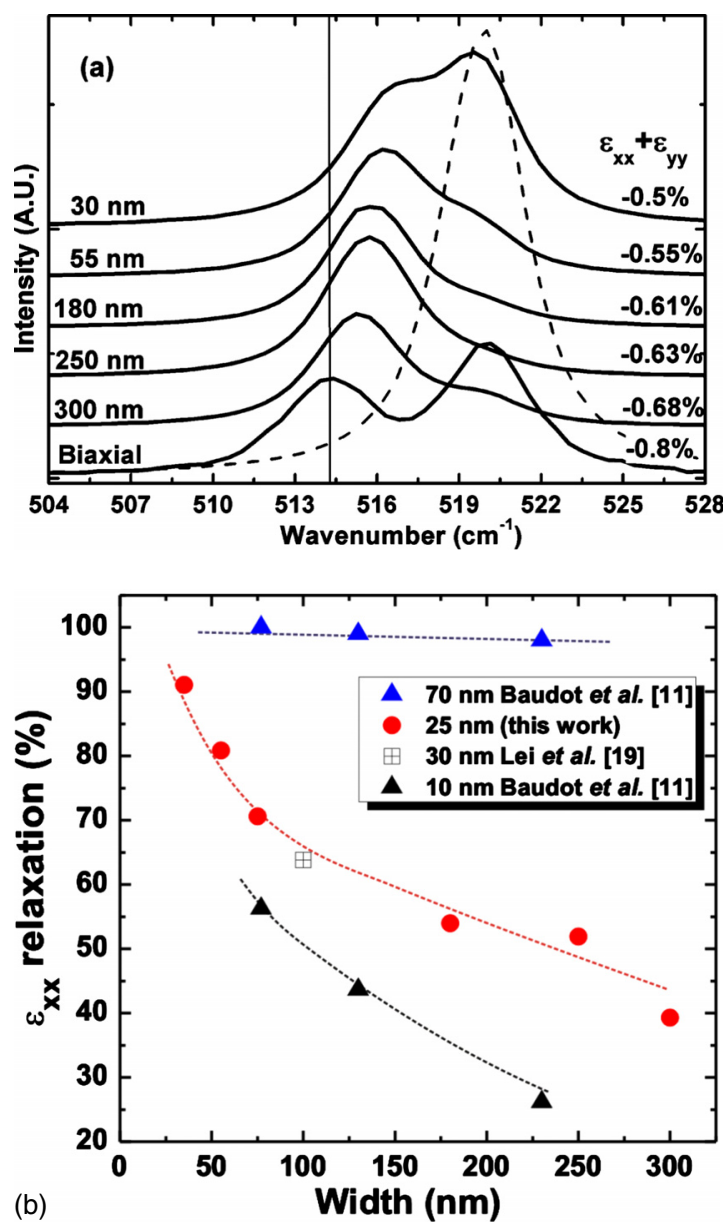

FIG. 6. (Color online) (a) Raman spectra of the SSOI layer and NWs of different widths. The strained Si peak shift toward to cubic Si peak as the width of the NW decreases; (b) the $\varepsilon_{x x}$ relaxation as function of NW width for different SSOI layer thicknesses. The relaxation is $100 \%$ for thick layer and decreases with the thickness of the SSOI layer.

After presenting the influence of NW patterning on the strain relaxation, we now investigate the influence of $\mathrm{As}^{+}$ion implantation and annealing on strained Si NWs.

\section{NW patterning of As implanted and annealed SSOI layers}

The NWs of this section were processed as described in Sec. II steming from both, doped and undoped SSOI layers. Doping was performed by implantation of $10 \mathrm{keV} \mathrm{As}{ }^{+}$ions to a dose of $2 \times 10^{15} \mathrm{~cm}^{-2}$ and subsequent $1000{ }^{\circ} \mathrm{C}$ spike annealing. According to Fig. 5 the strained Si peak shift, $\Delta \omega_{\mathrm{L}}$, between doped, $\omega_{\mathrm{L}-\mathrm{d}}$, and undoped, $\omega_{\mathrm{L}-\mathrm{u}}$, layers amounts to $\Delta \omega_{\mathrm{L}}=\omega_{\mathrm{L}-\mathrm{d}}-\omega_{\mathrm{L}-\mathrm{u}}=1.4 \mathrm{~cm}^{-1}$ Similarly, the peak shift due to doping effect for the $75 \mathrm{~nm}$ narrow NWs [Fig. 7(a)], $\Delta \omega_{\mathrm{NW}}$, between the doped, $\omega_{\mathrm{NW}-\mathrm{d}}$, and undoped one, $\omega_{\mathrm{NW}-\mathrm{u}}$ (measured in Sec. III B), is $\Delta \omega_{\mathrm{NW}}=\omega_{\mathrm{NW}-\mathrm{d}}-\omega_{\mathrm{NW}-\mathrm{u}}$ $=1.3 \mathrm{~cm}^{-1}$. This indicates the conservation of a majority of activated dopants after patterning of the highly doped SSOI layer. For the $35 \mathrm{~nm}$ narrow NWs fabricated on lower As doped layers, the shift in the Raman peak arising from dopant activation lies below the resolution limit of our Raman set-up, while a clear Raman shift was observed for the unpatterned SSOI layer. We attribute this to a loss in the dopant

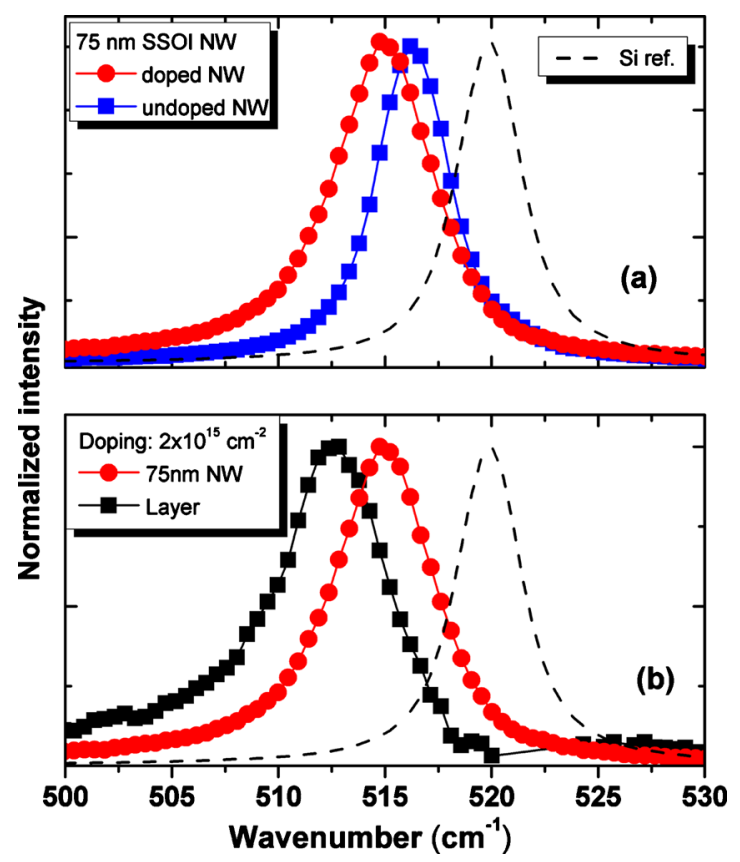

FIG. 7. (Color online) Raman spectra of (a) $75 \mathrm{~nm}$ narrow NWs unimplanted and implanted to $1 \times 10^{15} \mathrm{As}^{+} / \mathrm{cm}^{2}$ and (b) comparison of doped SSOI layer and doped $75 \mathrm{~nm}$ narrow NWs indicating strain relaxation after NWs patterning.

activation. Figure 7(b) compares the Raman spectra of a doped SSOI layer and $75 \mathrm{~nm}$ wide NWs fabricated on the same substrate indicating strain relaxation after NW patterning.

The electrical transport properties of the As doped NWs were determined using TLM method. Representative I-V curves for $75 \mathrm{~nm}$ narrow and $2 \mu \mathrm{m}$ long NWs implanted at different doses are plotted in Fig. 8, demonstrating the ohmic behavior of the doped NW.

Figure 9 displays the resistivity of NWs with different widths and the SSOI layer as a function of the implanted $\mathrm{As}^{+}$ dose. A dependence of the resistivity on the NW width and implantation dose is observed. For $75 \mathrm{~nm}$ narrow NWs, the resistivity is equivalent to the values of the layer, which is consistent with the Raman results presented in Fig. 7. The dose dependence becomes more pronounced for NW widths below $55 \mathrm{~nm}$. At the lower implantation dose of 5 $\times 10^{14} \mathrm{~cm}^{-2}$, the resistivity increases by a factor of 1.3 and 3.5 by decreasing the NW width from $55 \mathrm{~nm}$ to $35 \mathrm{~nm}$, respectively.

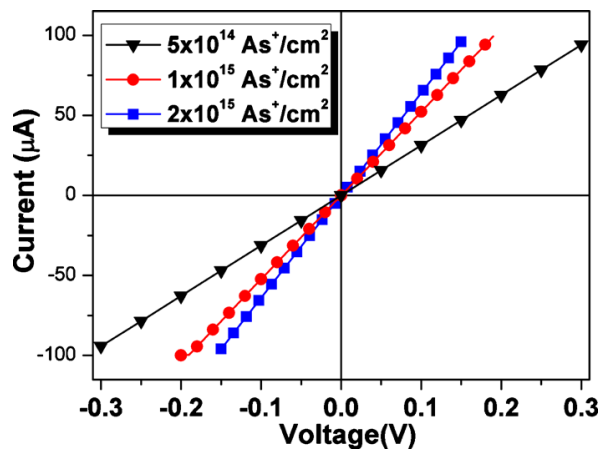

FIG. 8. (Color online) Current-voltage (I-V) measurements for a $75 \mathrm{~nm}$ narrow and $2 \mu \mathrm{m}$ long NWs implanted at different doses. 


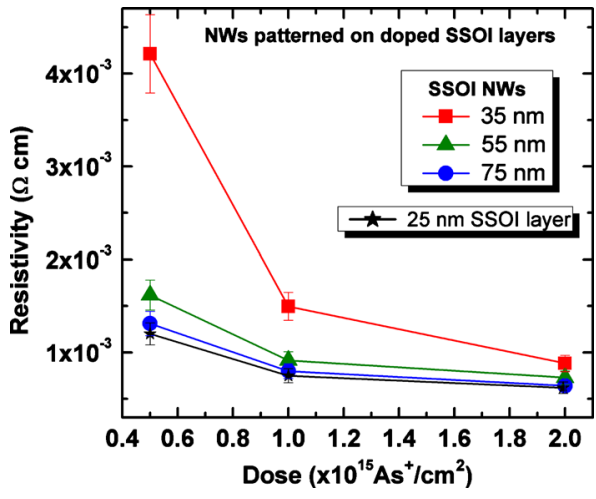

FIG. 9. (Color online) Resistivity of NWs fabricated on $25 \mathrm{~nm}$ SSOI doped layers as a function of implantation dose. The layer resistivity is added for comparison.

The observed increase in resistivity may be attributed to a series of effects such as quantum confinement, ${ }^{30}$ surface segregation of dopants, ${ }^{31}$ increased surface scattering due to larger surface area, size dependent strain relaxation and hence changed carrier mobilities ${ }^{26}$ and/or dielectric confinement effect. ${ }^{32}$ For the large range of NW widths used in this work, the quantum confinement effect is negligible because quantum confinement effects are expected for NW widths $<10 \mathrm{~nm}$. Since for NWs with equivalent width, respectively equivalent cross-section, the surface to volume ratio and the uniaxial strain relaxation are identical, we discard the possibility of surface scattering or strain change from biaxial to uniaxial as a driving phenomena affecting the NW resistivity. However, volume depletion due to impurity trapping at the NW surface, $\mathrm{Si} / \mathrm{SiO}_{2}$ interface in our case, becomes crucial, significantly reducing the active dopant concentration within the NW. If the surface trap concentration is assumed equal to the concentration of interface dangling bonds, typically of about $10^{12} \mathrm{~cm}^{-2}$, an estimated fraction of impurities of about $10^{19} \mathrm{~cm}^{-3}$ is neutralized. Above this impurity concentration, Coulomb screening is reduced and most impurities are ionized at room temperature. As a result, the depletion region most significantly affects the NW resistivity at low doping levels and very small NW dimensions, while it becomes small or even insignificant for heavily doped NWs, as seen in Fig. 9. If we assume that the carrier mobility is affected only by impurity scattering and is independent of the NW width, the activated dopant concentration $\left(N_{\mathrm{a}-\mathrm{NW}}\right)$ can be estimated from the measured NW resistivity $\left(\rho_{\mathrm{NW}}\right): N_{\mathrm{a}-\mathrm{NW}}$ $=1 /\left(\rho_{\mathrm{NW}} e \mu\right)$, where $e$ is the electronic charge and $\mu$ is the electron mobility in the SSOI layer of corresponding dopant concentration. The estimated dopant concentration in the $\mathrm{Si}$ NWs is displayed in Table II. For all implanted doses, the carrier concentration decreases nonlinearly with the width of the patterned structures, more pronounced for lower implantation doses. The ratio between the activated dopant concentrations in $35 \mathrm{~nm}$ narrow $\mathrm{NW}\left(N_{\mathrm{a}-35 \mathrm{NW}}\right)$ and in layer $\left(N_{\mathrm{a}-\mathrm{L}}\right)$ decreases from $N_{\mathrm{a}-35 \mathrm{NW}} / N_{\mathrm{a}-\mathrm{L}}=78 \%$ for $2 \times 10^{15} \mathrm{As}^{+} / \mathrm{cm}^{2}$ to $N_{\mathrm{a}-35 \mathrm{NW}} / N_{\mathrm{a}-\mathrm{L}}=30 \%$ for $5 \times 10^{14} \mathrm{As}^{+} / \mathrm{cm}^{2}$ indicating a dopant deactivation of about 2.7 times for the lower $\mathrm{As}^{+}$ion dose.

In conclusion, patterning of doped and annealed layers enables the fabrication of crystalline, strained and low resis-
TABLE II. Estimated dopant concentration in Si NWs as a function of the implantation dose calculated from the NW resistivity assuming equivalent mobility as in the SSOI layer.

\begin{tabular}{ccccc}
\hline \hline & \multicolumn{4}{c}{$\begin{array}{c}\text { Dopant concentration } N_{\mathrm{a}} \\
\left(\mathrm{cm}^{-3}\right)\end{array}$} \\
\cline { 2 - 5 } $\begin{array}{c}\text { Imp. dose } \Phi \\
\left(\mathrm{As}^{+} / \mathrm{cm}^{2}\right)\end{array}$ & Layer & $75 \mathrm{~nm} \mathrm{NW}$ & $55 \mathrm{~nm} \mathrm{NW}$ & $35 \mathrm{~nm} \mathrm{NW}$ \\
\hline $5 \times 10^{14}$ & $6.5 \times 10^{19}$ & $6.4 \times 10^{19}$ & $5.2 \times 10^{19}$ & $1.9 \times 10^{19}$ \\
$1 \times 10^{15}$ & $1.2 \times 10^{20}$ & $1.15 \times 10^{20}$ & $1.1 \times 10^{20}$ & $6.9 \times 10^{19}$ \\
$2 \times 10^{15}$ & $1.9 \times 10^{20}$ & $1.85 \times 10^{20}$ & $1.8 \times 10^{20}$ & $1.5 \times 10^{20}$ \\
\hline \hline
\end{tabular}

tivity NWs. Most notable are the results of the $35 \mathrm{~nm}$ wide uniaxially strained NWs, implanted at $2 \times 10^{15} \mathrm{As}^{+} / \mathrm{cm}^{2}$, exhibiting a resistivity close to that of the full layer.

\section{Directly implanted and annealed NWs}

Properties of ion implanted and annealed NWs can significantly differ from those patterned on doped layers. The critical issue is the effective width of the post-implantation seed available for SPER, which may affect the process of strain recovery as well as the formation of defect free crystalline structures. Additionally, dopant activation of implanted NWs may differ from NWs formed by patterning of doped layers. In this section, NWs were fabricated as described in Sec. II followed by $\mathrm{As}^{+}$ion implantation and a subsequent spike annealing.

The TEM image of Fig. 10 shows a cross-section of a NW implanted to $2 \times 10^{15} \mathrm{As}^{+} / \mathrm{cm}^{2}$ at $10 \mathrm{keV}$ and spike annealed at $1000{ }^{\circ} \mathrm{C}$. Although the NWs were partially amorphized after the implantation, Fig. 10 indicates that the nanostructure recrystallizes during spike annealing such that the center part of the wire is single crystalline, while the bottom edges, next to the buried $\mathrm{SiO}_{2}$ are polycrystalline.

Figure 11 compares the strain between the as-patterned and the implanted Si NWs. The as-patterned NWs show large strain relaxation $\left(\varepsilon_{x x}+\varepsilon_{y y}\right)$ up to $68 \%$ of the initial biaxially tensile strained SOI layer (Fig. 6). For the lowest implantation dose of $5 \times 10^{14} \mathrm{As}^{+} / \mathrm{cm}^{2}$, the annealed 55 and $75 \mathrm{~nm}$ NWs greatly recover the preimplantation strain state, while the $35 \mathrm{~nm}$ NWs relax by approximately $50 \%$. Figure 12(a) displays the deconvoluted strained Si signal from the Raman spectra of $35 \mathrm{~nm} \mathrm{NWs,} \mathrm{corresponding} \mathrm{to} \mathrm{the} \mathrm{as-}$ patterned and doped to $5 \times 10^{14} \mathrm{As}^{+} / \mathrm{cm}^{2} \mathrm{NWs}$. The shift in

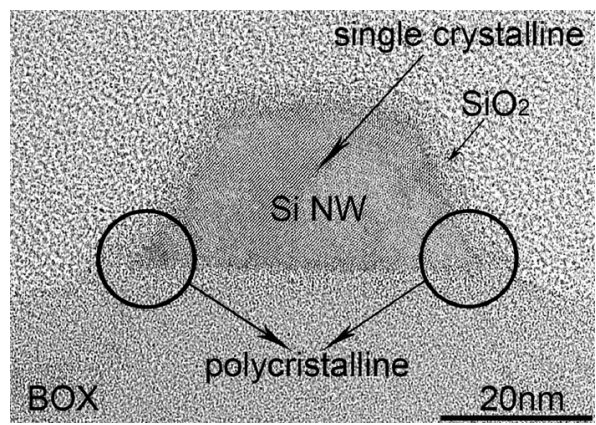

FIG. 10. XTEM image of a NW implanted to $2 \times 10^{15} \mathrm{As}^{+} / \mathrm{cm}^{2}$ and spike annealing showing the formation of a single crystal core and polygrains at the side edges of the NW. 


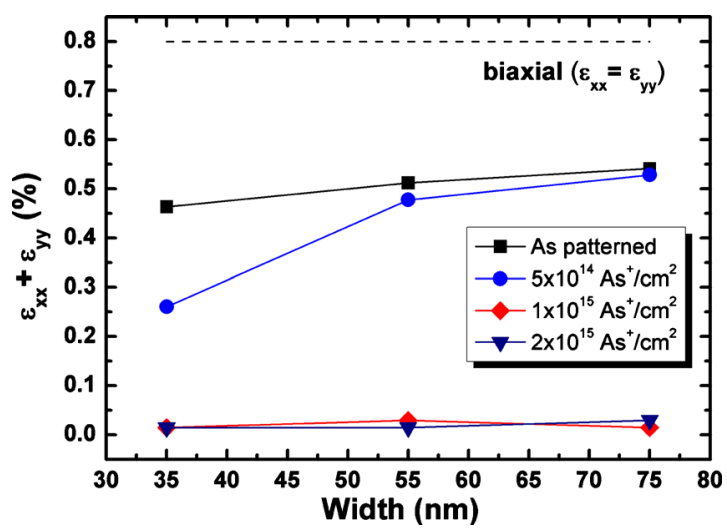

FIG. 11. (Color online) Sum of $\left(\varepsilon_{x x}+\varepsilon_{y y}\right)$ strain components of directly doped NWs as a function of NWs width for different implantation doses. The biaxial strain is indicated by the dashed line.

the strained Si peak of the doped NWs with respect to the as-patterned ones indicates strain relaxation while the symmetrical broadening of the peak indicates defective NW recrystallization.

At higher implantation doses, the recrystallization process results in full strain relaxation, $\varepsilon_{y y}=\varepsilon_{x x}=0$. Figure $12(\mathrm{~b})$ displays the Raman spectra of $75 \mathrm{~nm}$ wide NWs as-patterned (after doping to $2 \times 10^{15} \mathrm{As}^{+} / \mathrm{cm}^{2}$. The strain in the $\mathrm{NW}$ is
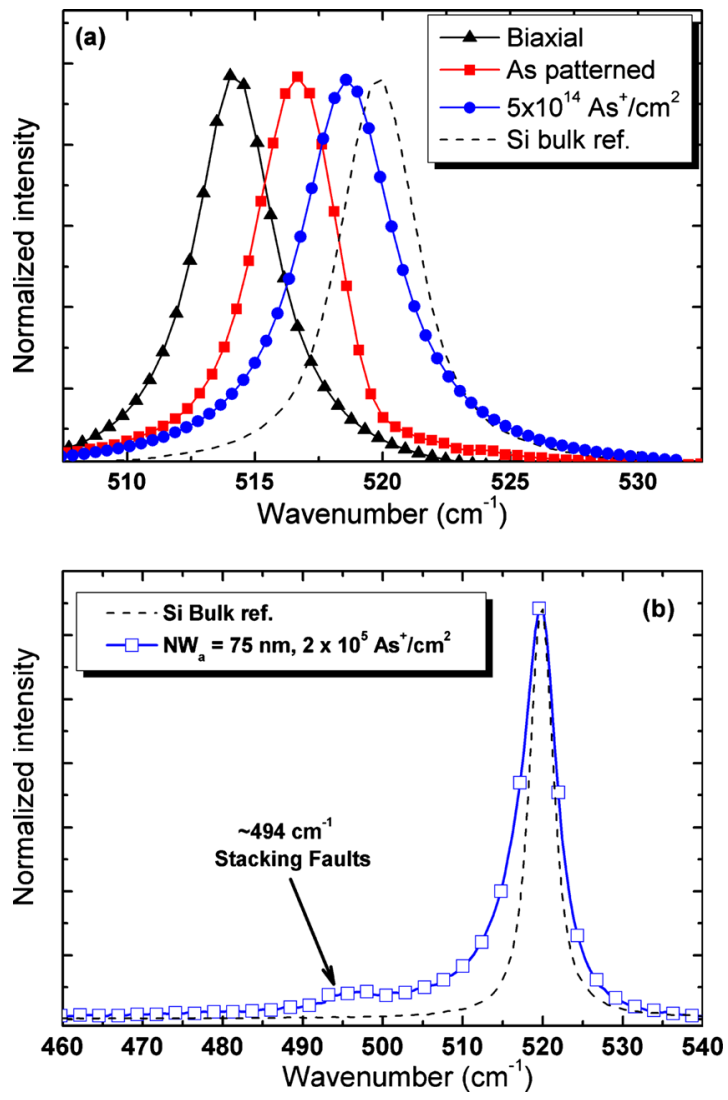

FIG. 12. (Color online) (a) The deconvoluted strained Si signal from the Raman spectra of $35 \mathrm{~nm}$ NWs corresponding to the as-patterned (square) and implanted to $5 \times 10^{14} \mathrm{As}^{+} / \mathrm{cm}^{2}$ spike annealed (circles) NWs. The signal from biaxial SSOI layer (triangles) and the cubic Si reference (dashed line) are added for comparison; (b) Raman spectra of a $75 \mathrm{~nm}$ wide NW recrystallized after ion implantation to $2 \times 10^{15} \mathrm{As}^{+} / \mathrm{cm}^{2}$ and spike annealing.

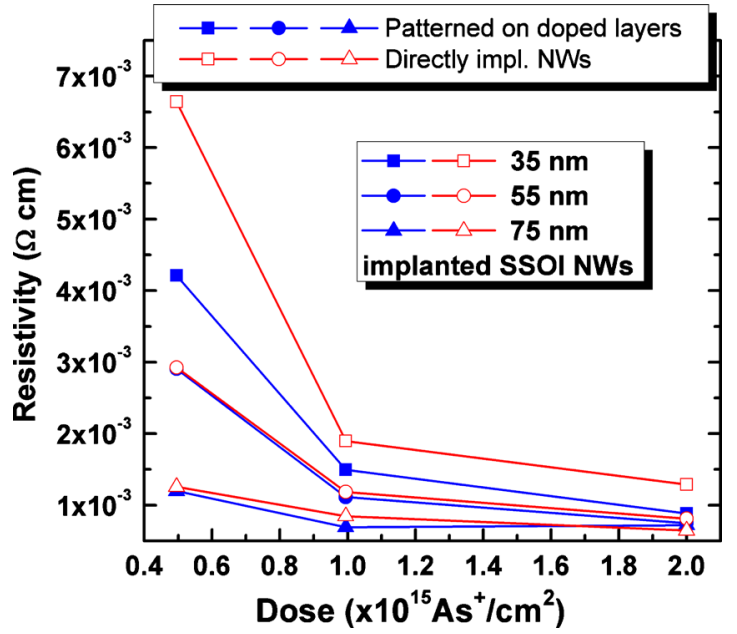

FIG. 13. (Color online) Comparison of Si NWs resistivity as a function of the implantation dose. Two types of doped NWs are presented: patterned on doped layers (full symbols) and implanted after patterning (empty symbols).

completely relieved after implantation and annealing, accompanied with a noticeable asymmetrical broadening of the peak attributed to the doping effect. The appearance of a small peak at $494 \mathrm{~cm}^{-1}$ is associated with the formation of stacking faults during NW recrystallization.

These observations indicate a very narrow parameter window for NW doping by ion implantation, which allows full strain recovery of the NWs. The use of tilted implantation results in amorphization of the sidewalls which also reduces the crystalline seed dimension. In consequence, the main difference between the layer recrystallization and NW is related to the size of the seed available for recrystallization: virtually infinite for the layer case (planar recrystallization in the $z$ direction) but finite for the NW structure (recrystallization in the $z-x$ directions) with possibly competing crystallization processes. Aside from recrystallization from the bottom to the top of the NW regrowth may start at various sites at the NW sidewalls. In the first case recrystallization yields to epitaxial regrowth and build up of elastic strain (see Fig. 11) while in the later case arbitrary nucleation, influenced by the NW walls crystalline direction and roughness, is likely to occur resulting in polycrystalline regions. At doses higher than $1 \times 10^{15} \mathrm{~cm}^{-2}$ the crystalline seed of the NW cannot be healed from the implantation defects and defective NWs are formed upon annealing. Indeed, Fig. 10 indicates the formation of a single crystal core and grains at the side edges of the NW. Therefore, the crystallization seed plays a key role on the quality of the recrystallized nanostructures, and is constrained by both, implantation dose and NW width.

The resistivity of the As implanted and annealed NWs as a function of implantation dose for different widths is plotted in Fig. 13. Similar to the NWs patterned on implanted layers, the $75 \mathrm{~nm}$ wide NWs show slight changes in resistivity as a function of the accumulated doses, with values around 1.2 $\times 10^{-3} \Omega \mathrm{cm}$. The dose dependence becomes evident for 55 $\mathrm{nm}$ NWs, particularly for the lower implantation dose of 5 $\times 10^{14} \mathrm{As}^{+} / \mathrm{cm}^{2}$ where the resistivity is remarkably higher than at higher doses. Generally, the $\rho_{\mathrm{NW}}$ is remarkably higher 
for directly implanted NWs than for NWs patterned from doped layers. As an example, the resistivity of a $55 \mathrm{~nm} \mathrm{NWs}$ implanted with $5 \times 10^{14} \mathrm{As}^{+} / \mathrm{cm}^{2}$ is up to 1.9 times higher than for implanted NWs.

For the directly implanted wires, an additional effect is considered to explain the lower resistivity of the NWs. Silvaco simulations of $10 \mathrm{keV} \mathrm{As}{ }^{+}$ion implantation of NWs under a tilt angle of $7^{\circ}$ indicate that the As atom concentration decreases strongly close to the NW lateral surfaces. We correlate this effect with $\mathrm{As}^{+}$ion loss during implantation due to straggling. SRIM simulations indicate a straggling length of $4.8 \mathrm{~nm}$, which is in accordance with the Silvaco simulation, where the As concentration varies by a factor of 2 within $5 \mathrm{~nm}$ from the NW sidewalls. ${ }^{33}$ Obviously, ion loss during implantation significantly impacts the resistivity as the width of the NWs and the implantation dose decrease. For both NW types where the surface to volume ratios are equivalent, the electronic transport degradation is also influenced by the lower crystallinity of directly implanted NWs.

\section{CONCLUSIONS}

We have presented the effects of $\mathrm{As}^{+}$ion implantation and annealing on the strain relaxation and resistivity of differently processed strained Si NWs. NWs with widths as small as $35 \mathrm{~nm}$ and $\mathrm{As}^{+}$ion implantation doses ranging from $5 \times 10^{14}$ to $2 \times 10^{15} \mathrm{As}^{+} / \mathrm{cm}^{2}$ were investigated. The studies revealed distinct differences of NWs patterned from previously doped layers as compared to undoped layers. Pseudomorphic epitaxial regrowth and concomitant complete strain recovery was observed for $25 \mathrm{~nm}$ thick SSOI layers, independent of the implanted dose in the investigated range. We found that strain conservation and low sheet resistivity close to the layer resistivity can be obtained only if the NWs are patterned from doped layers. Doping levels as high as 1.9 $\times 10^{20} \mathrm{~cm}^{-3}$ can be achieved using this approach. On the other hand, for NWs implanted and annealed after patterning, strain was maintained only for low $\mathrm{As}^{+}$implantation doses. The increase in the NW resistivity with decreasing width was attributed to dielectric confinement effect. Furthermore, the electrical resistivity of directly implanted NWs was generally higher than NWs patterned on doped layers. This was ascribed to a defective crystallization of NWs and ion loss during implantation due to straggling.

\section{ACKNOWLEDGMENTS}

The authors would like to thank Mr. W. Michelsen for performing the ion implantations, Mrs. Steffi Lenk for the electron microscopy, and Mr. M. Hülsbeck for the Raman spectroscopy measurements. This work was partially supported by the German Federal Ministry of Education and Research via the MEDEA+ project DECISIF (2T104) and by the European Community through Nanosil network of excellence (FP7 Grant No. 216171).
${ }^{1}$ K. Byon, D. Tham, J. E. Fischer, and A. T. Johnson, Appl. Phys. Lett. 90, 143513 (2007).

${ }^{2}$ R. S. Wagner and W. C. Ellis, Appl. Phys. Lett. 4, 89 (1964).

${ }^{3}$ M. L. Lee, E. A. Fitzgerald, M. T. Bulsara, M. T. Currie, and A. Lochetfeld, J. Appl. Phys. 97, 011101 (2005).

${ }^{4}$ K.-W. Ang, J. Lin, C.-H. Tung, N. Balasubramanian, G. S. Samudra, and Y.-C. Yeo, IEEE Trans. Electron Devices 55, 850 (2008).

${ }^{5}$ S. F. Feste, J. Knoch, S. Habicht, D. Buca, Q. T. Zhao, and S. Mantl, Proceedings of the Tenth International Conference on Ultimate Integration of Silicon ULIS, Aachen, 18-20 March 2009, p. 109.

${ }^{6}$ T. Irisawa, T. Numata, T. Tezuka, N. Sugiyama, and S. Takagi, Tech. Dig. - Int. Electron Devices Meet. 2006, 457.

${ }^{7}$ G. M. Cohen, S. Bangsaruntip, S. Laux, M. J. Rooks, J. Cai, and L. Gignac, 66th Device Research Conference Digest, Santa Barbara, 23-25 June 2008, p. 187.

${ }^{8}$ J.-P. Colinge, C.-W. Lee, A. Afzalian, N. D. Akhavan, R. Yan, I. Ferain, P. Razavi, B. O'Neill, A. Blake, M. White, A.-M. Kelleher, B. McCarthy, and R. Murphy, Nat. Nanotechnol. 5, 225 (2010).

${ }^{9}$ T. S. Drake, C. Ni Chleirigh, M. L. Lee, A. J. Pitera, E. A. Fitzgerald, D. A. Antoniadis, D. H. Anjum, J. Li, R. Hull, N. Klymko, and J. L. Hoyt, Appl. Phys. Lett. 83, 875 (2003).

${ }^{10}$ A. Wai, S. Duenkel, R. Boschke, and M. Horstmann, ECS Trans. 6(1), 15 (2007).

${ }^{11}$ S. Baudot, F. Andrieu, F. Rieutord, and J. Eymery, J. Appl. Phys. 105, 114302 (2009).

${ }^{12}$ N. Singh, K. D. Buddharaju, S. K. Manhas, A. Agarwal, S. C. Rustagi, G. Q. Lo, N. Balasubramanian, and D.-L. Kwong, IEEE Trans. Electron Devices 55, 3107 (2008).

${ }^{13}$ B. Ghyselen, J. M. Hartmann, T. Ernst, C. Aulnette, B. Osternaud, Y. Bogumilowicz, A. Abbadie, P. Besson, O. Rayssac, A. Tiberj, N. Daval, I. Cayrefourq, F. Fournel, H. Moriceau, C. Di Nardo, F. Andrieu, V. Paillard, M. Cabie, L. Vincent, E. Snoeck, F. Cristiano, A. Rocher, A. Ponchet, A. Claverie, P. Boucaud, M. N. Semeria, D. Bensahel, B. Kernevez, and C. Mazure, Solid-State Electron. 48, 1285 (2004).

${ }^{14}$ J. F. Ziegler, J. P. Biersack, and U. Littmark, The Stopping Range of Ions in Solids (Pergamon, New York, 1985) and SRIM-The Stopping and Range of Ions in Matter http://www.srim.org/

${ }^{15}$ R. H. Dennard, F. H. Gaensslen, H. N. Yu, V. L. Rideout, E. Bassous, and A. R. LeBlanc, IEEE J. Solid-State Circuits 9, 256 (1974).

${ }^{16}$ P. Dobrosz, S. J. Bull, S. H. Olsen, and A. G. O'Neill, Surf. Coat. Technol. 200, 1755 (2005).

${ }^{17}$ V. Vartanian, S. Zollner, A. V.-Y. Thean, T. White, B.-Y. Nguyen, L. Prabhu, D. Eades, S. Parsons, H. Desjardins, K. Kim, Z.-X. Jiang, V. Dhandapani, J. Hildreth, R. Powers, G. Spencer, N. Ramani, M. Kottke, M. Canonico, X.-D. Wang, L. Contreras, D. Theodore, R. Gregory, and S. Venkatesan, IEEE Trans. Semicond. Manuf. 19, 381 (2006).

${ }^{18}$ R. Z. Lei, W. Tsai, I. Aberg, T. B. O'Reilly, J. L. Hoyt, D. A. Antoniadis, H. I. Smith, A. J. Paul, M. L. Green, J. Li, and R. Hull, Appl. Phys. Lett. 87, 251926 (2005).

${ }^{19}$ D. K. Schroder, Semiconductor Material and Device Characterization, 3rd ed. (Wiley, New York, 2006), p. 138.

${ }^{20}$ N. Clément, D. Tonneau, H. Dallaportaa, V. Bochiat, D. Fraboulet, D. Mariole, J. Gautier, and V. Safarov, Physica E 13, 999 (2002).

${ }^{21}$ D. Buca, R. A. Minamisawa, H. Trinkaus, B. Holländer, S. Mantl, R. Loo, and M. Caymax, J. Appl. Phys. 105, 114905 (2009).

${ }^{22}$ W. Heiermann, D. Buca, H. Trinkaus, U. Breuer, B. Ghyselen, and M. Mantl, ECS Trans. 19(1), 95 (2009).

${ }^{23}$ F. Cerdeira and M. Cardona, Phys. Rev. B 5, 1440 (1972).

${ }^{24}$ L. O'Reilly, K. Horan, P. J. McNally, N. S. Bennett, N. E. B. Cowern, A. Lankinen, B. J. Sealy, R. M. Gwilliam, T. C. Q. Noakes, and P. Bailey, Appl. Phys. Lett. 92, 233506 (2008).

${ }^{25}$ O. Moutanabbir, M. Reiche, W. Erfurth, F. Naumann, M. Petzold, and U. Gösele, Appl. Phys. Lett. 94, 243113 (2009).

${ }^{26}$ S. F. Feste, J. Knoch, S. Habicht, D. Buca, Q. T. Zhao, and S. Mantl, Solid-State Electron. 53, 1257 (2009).

${ }^{27}$ J. L. Hoyt, P. Hashemi, and L. Gomez, ECS Trans. 16(10), 731 (2008).

${ }^{28}$ S. F. Feste, S. Habicht, Q. T. Zhao, D. Buca, and S. Mantl, Proceeding of the 39th European Solid-State Device Research Conference (ESSDERC), Athens, Greece, 14-18 September 2009, p. 323.

${ }^{29}$ J. He, W. L. Morris, M. C. Shaw, J. C. Mather, and N. Sridhar, Int. J. Microcircuits Electron. Packag. 21, 297 (1998).

${ }^{30}$ D. R. Khanal, J. W. L. Yim, W. Walukiewicz, and J. Wu, Nano Lett. 7, 1186 (2007).

${ }^{31}$ M. V. Fernández-Serra, C. Adessi, and X. Blase, Phys. Rev. Lett. 96, 
166805 (2006)

${ }^{32}$ M. T. Björk, H. Schmid, J. Knoch, H. Riel, and W. Riess, Nat. Nanotechnol. 4, 103 (2009).

${ }^{33}$ S. Bangsaruntip, G. M. Cohen, M. Laurent, M. Bjoerk, H. Ghoneim, H.
Schmid, and H. Riel, Oral presentation, Symposium P, Session P8, Material Research Society (MRS) Spring Meeting, April 5-9 2010, San Francisco, California, MRS Symposia Proceedings (Materials Research Society, Pittsburgh, 2010), p. P8.5. 